\title{
JUURNAL.RU
}

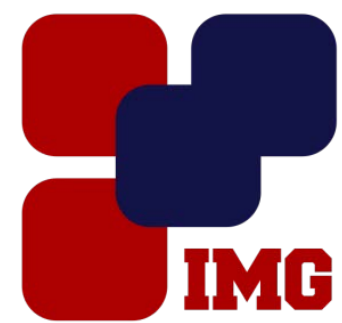
IYANOY Management GROUP

Анохина Е.C. Наииональный исследовательский ядерный университет «МИФИ» Москва, Россия

doi: 10.18411/lj-31-03-2017-1-12

idsp 000001:lj-31-03-2017-1-12

\section{Формирование портфелей проектов на основе нечётких моделей}

\section{Аннотация}

Данная статья посвящена проблемам формирования портфеля проектов в условиях неопределённости. В работе излагается экономический смысл показателей эффективности проекта, таких как NPV, IRR, срока окупаемости и целесообразность инвестирования капитала в проект. Также рассматривается математическая модель нахождения оптимального решения многокритериальных задач среди множества решений Парето. Освящается вопрос применения теории нечётких множеств при решении задачи оптимального распределения капитала между объектами инвестирования. Описывается математическая модель формирования эффективных инвестиционных портфелей с использованием трапециевидных нечетких чисел. Также работа содержит обоснование использования метода, основанного на нечетко-множественном подходе, для оценки инвестиционных проектов, как наиболее эффективного в условиях риска и неопределенностей. Гибкость и мощность методов теории нечётких множеств позволяют рассматривать их как перспективное и эффективное средство для решения различных задач управления проектами.

Ключевые слова: оценка, инвестиционный проект, эффективность, чистый дисконтированный доход, внутренняя норма доходности, дисконтированный период окупаемости, решения Парето, портфель проектов, нечеткие трапециевидные числа.

\section{Abstract}

The article deals with the problems of formation of a portfolio of projects under uncertainty.Showed economic sense indicators NPV, IRR, and payback period of capital investment feasibility of the project.A mathematical model for finding the optimal solution of multicriteria tasks among multiplePareto solutions. Sanctified question of the application of the theory of fuzzy sets to solve the problem of optimal allocation of capital investment between objects.A mathematical model of efficient investment 
portfolios using the trapezoidal fuzzy numbers. Also, the article contains a rationale for using a method based on a fuzzy-multiple approach to evaluate investment projects, as the most effective in terms of risk and uncertainty. The flexibility and power of the methods of the theory of fuzzy sets allow us to consider them as a promising and effective tool for solving various problems of project management.

Keywords: evaluation, investment project, efficiency, net present value, internal rate of return, discounted payback period, the Pareto solutions portfolio, trapezoidal fuzzy number.

Проблема формирования портфеля проектов относится к задачам оптимизации в условиях неопределенности. Как правило, для решения подобных задач привлекается аппарат теории вероятности. Однако, в ряде ситуаций применение теории вероятности представляется недостаточно корректным и обоснованным. Причина этому - недостаток имеющихся данных, что не позволяет с достаточной степенью уверенности установить адекватность выбранной для описания ситуации вероятностной модели. Если в задаче формирования портфеля инвестиций в ценные бумаги к услугам аналитика предоставляются массивы котировок финансовых инструментов, охватывающие месяцы и годы и позволяющие использовать всю мощь статистического анализа, то при рассмотрении реальных инвестиций основным, но весьма ограниченным источником информации о риске являются экспертные оценки. В таких условиях появляется потребность в других, отличных от вероятностного, подходах к оценке имеющейся неопределенности. Один из таких подходов основан на применении теории нечётких множеств. С применением данной методики появляется возможность свести качественные экспертные оценки к количественным, числовым (нечётким). С другой стороны, нечёткие множества предоставляют эксперту большую гибкость при оценивании численных показателей.

Портфельное инвестирование, вообще говоря, является одним из самых перспективных направленийв экономической. Основы современной теории портфельного инвестирования были заложены в 50-е годы в работах американского математика-экономиста Г.Марковица. В настоящее время теория портфельного инвестирования широко используется в странах с развитой рыночной экономикой и является одним из основных инструментов, с помощью которых повышается эффективность материальных и финансовых ресурсов. Создание математической модели формирования эффективных портфелей в условиях неопределенности обусловлена возможностью использования разработанной математической модели для решения задач эффективного распределения ресурсов в различных конкретных секторах отечественной экономики. Известно, что оптимальное решение многокритериальных задач необходимо искать среди множества решений Парето, обладающих тем свойством, что улучшение любого критерия для решения Парето приводит к ухудшению других критериев. Множество решений Парето для двухкритериальной задачи можно найти, фиксируя один из критериев и 
оптимизируя другой. В этом случае кроме решений Парето могут появиться, не обладающие свойством Парето, но такие «лишние» решения легко выделить и исключить. Согласно схеме Марковица, для нахождения решений Парето двухкритериальной задачи оптимизации портфеля инвестиций осуществляется минимизация риска портфеляб $(\vec{x})$ при фиксированных значениях ожидаемого среднего значения $\mathrm{m}_{0}(. \vec{x})$.

Для решения задач оптимального распределения капитала между рассматриваемыми объектами инвестирования согласно схеме Марковица достаточно знать лишь две характеристики случайного вектора эффективностей вектор математических ожиданий и ковариационную матрицу W. Математическое предположение о наличии неопределенности означает, что Ri - случайные величины.

$$
\text { Wij }=\operatorname{cov}(\mathrm{Ri}, \mathrm{Rj}), \mathrm{i}, \mathrm{j}=1, \ldots, \mathrm{n}(1)
$$

Математическая модель оптимизации портфеля инвестиций, согласно схеме Марковица, принадлежит к классу задач квадратичного программирования, поскольку минимизируется квадратичная форма $(\mathrm{W} \vec{x}, \vec{x})$. при линейных ограничениях типа равенств и неравенств. Портфель характеризуется двумя критериями - ожидаемым средним значением эффективности портфеля $\mathrm{m}_{\mathrm{e}}=\mathrm{m}_{\mathrm{o}}(\vec{x})$ и риском портфеля $\sigma_{\varrho}=\sigma_{\varrho}(\vec{x})$, оба критерия зависят от выбранного состава портфеля $\vec{x}$. Критерий $\mathrm{m}_{\varrho}(\vec{x})$. необходимо увеличить, а критерий $\sigma_{0}$ уменьшить, изменяя состав портфеля (вектор $\vec{x})$. Согласно Марковицу, можно в качестве риска выбирать дисперсию эффективности портфеля или риск портфеля $\sigma_{\varrho}^{2}$

$$
\mathrm{m}_{\mathrm{Q}}=\sum_{i=1}^{n} x_{i} * m_{i}=(\vec{x}, \vec{m})(2)
$$

Очевидно, $\mathrm{m}_{. \varrho}$ принадлежит промежутку $\left[m_{\min }, m_{\max }\right]$, где $m_{\min }=\min \{\mathrm{m} 1, \ldots, \mathrm{mn}\},. m_{\max } .=\max \{\mathrm{m} 1, \ldots, . \mathrm{mn}\}$. $\sigma_{\mathrm{Q}}^{2}=\sum_{i=1}^{n} \times \sum_{j=1}^{n} x_{i} \times x_{j} \times W_{i j}=(\mathrm{W} \vec{x}, \vec{x})(3)$

Для того чтобы избежать посторонних непаретовских решений, необходимо решить задачу формирования портфеля с минимально возможным значением риска , математическая модель которой имеет вид

$$
\begin{aligned}
& \sigma_{Q}{ }^{2}=(\mathrm{W} \vec{x}, \vec{x})-\min . \\
& x_{i} \geq 0, \mathrm{i}=1, \ldots, \mathrm{n} \sum_{i=1}^{n} x_{i}=1 .
\end{aligned}
$$

Наличие риска подразумевает наличие неопределенности. Нечеткие множества допускают промежуточные степенные принадлежности, в этом состоит их принципиальное отличие от обычных (или четких) множеств. Применение аппарата нечеткой логики, в частности использование трапециевидных чисел, упрощает решение задач формирования эффективных инвестиционных портфелей в условиях неопределенности. Общепризнанными показателями, характеризующими инвестиционный проект, служат такие величины, как чистый дисконтированный доход NPV, внутренняя норма доходности IRR, срок окупаемости. При положительном значении NPV вложение капитала в рассматриваемый проект является эффективным (прибыльным), а при отрицательном NPV - неэффективным (убыточным). Для того что на практике 
инвестирование имело экономико-финансовый смысл необходимо, что бы NPV было положительным. Положительность NPV является необходимым условием для участия инвестора в инвестировании средств в рассматриваемый объект (проект). Если NPV $>0$, то условия инвестирования являются предварительно приемлемыми для инвестора (с точки зрения NPV). Экономический смысл IRR заключается в том, что он показывает максимальную норму доходности, то есть определяет максимальную стоимость привлекаемого капитала, при которой инвестиционный проект остается выгодным. Считается, что чем больше положительная разница IRR-p, тем устойчивее проект. Целесообразно инвестировать капитал в проект, когда NPV=0 при $\mathrm{p}=\mathrm{IRR},(5)$

где $\mathrm{p}$ - это процентная ставка.

Инвестиции это всегда риск, но риск можно уменьшить, и использование нечёткой логики, в частности, привлечение аппарата нечётких чисел в решении задачи распределения ресурсов, значительно снижает риск. Применение аппарата нечеткой логики, в частности использование трапециевидных чисел, упрощает решение задач формирования эффективных инвестиционных портфелей в условиях неопределенности.

При вычислении каждого из общепринятых показателей эффективности денежный поток проекта предполагается известным. Однако реальность такова, что, как правило, невозможно получить точную оценку потока проекта. В этом случае удобно использовать нечёткие числа, параметры которых могут быть оценены экспертами. Например, при ответе на вопрос, каким будет ожидаемый доход от проекта, эксперт может указать пессимистическую $d_{\text {песс }}$, оптимистическую $d_{\text {опт }}$ и наиболее вероятную $d_{\text {вер }}$ оценки, и полученную информацию можно объединить в виде нечёткого треугольного числа $\mathrm{D}=\left(d_{\text {песс }}\right.$, $\left.d_{\text {вер}}, d_{\text {опт }}\right)$. Денежный поток проекта задаётся как набор трапециевидных нечётких чисел $C_{t}=\left(c_{t 1}, c_{t 2}, c_{t 3}, c_{t 4}\right), \quad \mathrm{t}=0,1,2,3, \ldots, \mathrm{T}$.

Число $c_{t 1}$ интерпретируется как наименьшее возможное значение потока в момент времени $t$, поток ни при каких обстоятельствах не может опускаться ниже этого значения, $c_{t 4}$ - наибольшее возможное значение, а числа $c_{t 2}$ и $c_{t 3}$ образуют интервал в пределах которого, скорее всего, будет находиться значение денежного потока. Довольно часто для оценки используют треугольные нечёткие числа $C_{t}=\left(c_{t 1}, c_{t 2}=c_{t 3}, c_{t 4}\right)$, при этом число $c_{t 1}$ есть пессимистическая, $c_{t 4}-$ оптимистическая, а $c_{t 2}$ - наиболее вероятная оценка денежного потока проекта (Рис.1).

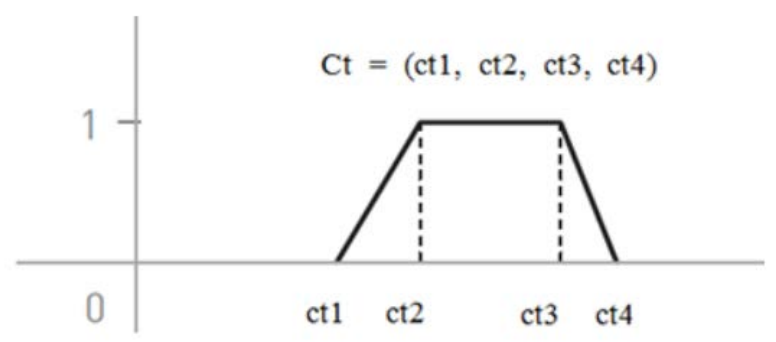

Рисунок 1. Трапециевидное число 
Аналогичным образом ставка дисконтирования также представляется в виде нечёткого числа $\mathrm{r}=(\mathrm{r} 1, \mathrm{r} 2, \mathrm{r} 3, \mathrm{r} 4)$. Чтобы найти выражение для нечёткого NPV, нужно, как и в обычном случае, суммировать (нечёткие) дисконтированные значения для всех компонент денежного потока: $N P V=\sum_{t=0}^{T} P V\left(C_{t}\right)$.

$\mathrm{B}$ свою очередь дисконтированное значение $P V\left(C_{t}\right)$ получается применением принципа расширения к классической формуле: $\quad P V\left(C_{t}\right)=\frac{\left(C_{t}\right)}{(1+r)^{t}}(7)$

$\mathrm{B}$ итоге получаем дисконтированный чистый денежный поток в момент t:

$$
P V\left(C_{t}\right)=\left(\begin{array}{l}
\frac{\max \left(c_{t 1}, 0\right)}{\left(1+r_{4}\right)^{t}}+\frac{\min \left(c_{t 1}, 0\right)}{\left(1+r_{1}\right)^{t}}, \\
\frac{\max \left(c_{t 2}, 0\right)}{\left(1+r_{3}\right)^{t}}+\frac{\min \left(c_{t 2}, 0\right)}{\left(1+r_{2}\right)^{t}}, \\
\frac{\max \left(c_{t 3}, 0\right)}{\left(1+r_{2}\right)^{t}}+\frac{\min \left(c_{t 3}, 0\right)}{\left(1+r_{3}\right)^{t}}, \\
\frac{\max \left(c_{t 4}, 0\right)}{\left(1+r_{1}\right)^{t}}+\frac{\min \left(c_{t 4}, 0\right)}{\left(1+r_{4}\right)^{t}}
\end{array}\right) .
$$

Подставляя полученное выражение в предыдущую формулу (6), нетрудно получить формулу для чистой текущей стоимости проекта:

$$
\mathrm{NPV}=\left(\sum_{t=0}^{T} d_{t 1}, \sum_{t=0}^{T} d_{t 2}, \sum_{t=0}^{T} d_{t 3}, \sum_{t=0}^{T} d_{t 4}\right)
$$

где $P V\left(C_{t}\right)=\left(d_{t 1}, d_{t 2}, d_{t 3}, d_{t 4}\right)$

Внутренняя норма доходности проекта с нечёт-ким денежным потоком вычисляется по формуле: IRR $=\left(i r r_{1}, i r r_{2}, i r r_{3}, i r r_{4}\right)$,

где: $\operatorname{irr}_{k}(\mathrm{k}=1,2,3,4)$ - внутренняя норма доходности проекта с (чётким) денежным потоком $c_{0 k}, c_{1 k}, \ldots, c_{T k}$, таким образом, $\operatorname{irr}_{k}$ есть корень уравнения:

$$
\sum_{t=0}^{T} \frac{\left(C_{t k}\right)}{\left(1+i r_{k}\right)^{t}}=0
$$

Точно так же срок окупаемости представляется в виде трапециевидного числа:

$$
\mathrm{PP}=(\mathrm{p} 1, \mathrm{p} 2, \mathrm{p} 3, \mathrm{p} 4)
$$

где: $P_{k}$ - срок окупаемости проекта с потоком $c_{0 k}, c_{1 k}, \ldots, c_{T k}$. Таким образом: $P_{k}=\min _{p}\left\{p: \sum_{t=0}^{[p]} d_{t k}+(p-[p]) d_{[p]+1, k} \geq 0\right\}$ где [р] - целая часть числа р и $P V\left(C_{t}\right)=\left(d_{t 1}, d_{t 2}, d_{t 3}, d_{t 4}\right)$.

Применение теории нечётких множеств открывает новые методы и возможности для решения задач оценивания проектов и формирования оптимального портфеля проектов. Нечёткие множества позволяют учитывать качественные характеристики проектов, преобразуя их в численный вид, применительно к количественным характеристикам проекта, таким как NPV, теория предоставляет средства для работы с неопределённостью даже в тех случаях, когда имеющейся информации недостаточно, чтобы делать статистические выводы с необходимым уровнем достоверности. Гибкость и мощность методов теории нечётких множеств позволяют рассматривать их как перспективное и эффективное средство для решения различных задач управления проектами [1,2]. 


\section{Литература}

1. Авдошин С.М., Лифшиц А.А. Формирование портфеля проектов на основе нечеткой модели многокритериальной оптимизации. Бизнес информатика №1(27) -2014, с. 15-19

2. Аньшин В.М., Демкин И.В., Царьков И.Н., Никонов И.М. Применение теории нечётких множеств к задаче формирования портфеля проектов. Проблемы анализа риска, Том 5, 2008, № 3 с. 9-13 